\title{
Kernos
}

Revue internationale et pluridisciplinaire de religion grecque antique

8| 1995

Varia

\section{Note sur le Sépias. Mythe et histoire}

\section{Philippe Borgeaud}

URL : http://journals.openedition.org/kernos/583

DOI : $10.4000 /$ kernos.583

ISSN : 2034-7871

\section{Éditeur}

Centre international d'étude de la religion grecque antique

\section{Édition imprimée}

Date de publication : 1 janvier 1995

Pagination : 23-29

ISSN : 0776-3824

Référence électronique

Philippe Borgeaud, « Note sur le Sépias. Mythe et histoire », Kernos [En ligne], 8| 1995, mis en ligne le 11 avril 2011, consulté le 01 mai 2019. URL : http://journals.openedition.org/kernos/583 ; DOI :

10.4000/kernos.583 


\section{Note sur le Sêpias. Mythe et histoire}

Le scholiaste au vers 582 du chant I des Argonautiques d'Apollonios propose du Sépias la définition suivante: Sépias, promontoire (akroterion) de Magnésie, ainsi appelé parce que Thétis, en ce lieu, se métamorphosa en seiche (sepia) alors qu'elle était poursuivie par Pélée. Dans le Banquet des sophistes (I, 30d), Athénée rapporte ce même épisode saugrenu, en ajoutant que les seiches abondent dans la région du Sépias (qui tire certainement son nom de l'animal).

La métamorphose en seiche de la néréide Thétis, sombre déesse des profondeurs marines, s'explique par les qualités remarquables du mollusque : "nœud vivant de liens mobiles et animés " ${ }^{1}$, dont la couleur blanche évoque pour les Grecs le teint pâle d'une peau préservée du soleil (l'idéal de la beauté féminine), la seiche " porte en elle un liquide noir, le tholos. Quand elle émet cette encre, elle crée autour d'elle une obscurité impénétrable au sein de laquelle elle se dissimule, un nuage de nuit où se brouillent et se confondent toutes les routes de la mer ${ }^{2}$. La belle déesse tente d'échapper à l'étreinte du mortel. En vain. Sa ruse et ses métamorphoses (analogues à celles de Protée, et bien connues par l'iconographie des vases attiques dès le début du Ve siècle) ne serviront à rien. Zeus et Poséidon ont voulu cette union, après avoir renoncé à se disputer eux-mêmes les faveurs de la fille de Nérée; ils ont appris en effet, de Thémis ou de Prométhée, que le fils de Thétis et d'un immortel serait plus puissant que son père et le détrônerait. La Terre de son côté, alourdie par la prolifération des humains, ourdissait sa ruse, préparant l'enchaînement des causes qui conduiront à la guerre de Troie, terme de la race des héros. Les Immortels ont donc décidé ce mariage, et la foule des dieux se rend aux noces de Pélée et de Thétis. La tradition situe la scène du mariage en Thessalie, près de Pharsale, où s'élevait un sanctuaire de Thétis, le Thétideion, distinguant ainsi les noces fameuses (on y verra jetée la pomme de discorde, origine de la guerre de Troie, sinistre présage de l'enfant à naître, Achille...), de la scène érotique, marine, où Pélée s'empare de Thétis et s'unit pour la première fois à elle. Cette scène-là est localisée dans la région du Sépias, sur les côtes sud-est de la péninsule de Magnésie, à proximité de l'île de Skiathos. Un promontoire

1 M. DETIENNE et J.-P. VERNANT, Les Ruses de l'intelligence, Paris, Flammarion, 1974, p. 160.

2 Ibid., p. 163. 
où étaient passés les Argonautes ${ }^{3}$, situé non loin de la halte des Aphètes (halte que les Anciens pouvaient localiser soit dans la région de Trikeri, soit sur les plages de Platania). La région du Sépias correspond donc très précisément au cap qui sépare l'actuel petit port de Katigiorgis et le hameau de Mourtias, sur le territoire de Liri (une région où les traces d'occupation antique ne sont pas inconnues ${ }^{4}$ ).

Le pli profond de l'antique roc de Sépias ${ }^{5}$ est donc le lieu des amours de Pélée et de Thétis. C'est aussi le lieu où Thétis ordonne au vieux Pélée de se rendre, au moment du malheur le plus profond, pour attendre le cortège des cinquante Néréides qui viendront, en compagnie de sa divine épouse, l'entrâ̂ner vers l'immortalité marine. Devenu compagnon de Nérée, Pélée divinisé pourra communiquer, du Sépias, par les routes sous-marines, avec le nord de la Mer Noire où son fils Achille, enlevé lui aussi au sort commun des mortels, séjourne sur l'île Blanche :

Thétis :

Pélée, je suis Thétis, et je viens à cause de notre mariage d'autrefois. J'ai quitté la maison de Nérée... Il faut que tu saches ce que tu retires de mon amour, car je suis déesse par ma naissance, et je suis l'enfant d'un père qui est dieu. Je te délivrerai des malheurs de la condition d'homme en faisant de toi un immortel et un dieu sans déclin. Après cela, dans la maison de Nérée, avec moi, pour le temps à venir, tu habiteras, comme un dieu habite avec une déesse. Alors tu sortiras de la mer, le pied sec. Tu iras voir mon fils et le tien, Achille, notre Bienaimé, qui habite un palais sur une île de la Côte Blanche, au-delà du détroit hospitalier... Reviens t'installer dans le pli profond de l'antique Roc de Sépias. Attends-moi là jusqu'au moment où tu me verras sortir des eaux avec le chœur des cinquante Néréides pour t'escorter. Selon l'arrêt du destin, il faut t'emmener au loin. Zeus en a décidé ainsi, Cesse de pleurer sur les morts.

3 Ils longeaient les falaises du Pélion en voguant toujours de l'avant; le cap Séplas (Sepias akre) s'effaçatt à l'bortzon; Skiatbos apparaissatt au milleu de la mer: APOLL. RHODES, Argonautiques, I, 581-582 (trad. Delage). Cf. infra, n. 11.

4 Cf. l'article Seplas, R.-E. (STAEHLIN), qui renvoie à MEzières, Mémotre sur le Pélion et l'Ossa, p. 210 sq.; A.J.B. WACE et J.P. DROOP, Excavations at Theotokou, Thessaly, in ABSA, 13 (1906-1907), p. 309-327 et pl. X-XI, ont dégagé, légèrement en retrait de la plage où s'élève la petite chapelle de la Theotokos (élevée au XIXe s., en partie avec des remplois antiques), à la sortie du village de Liri, les vestiges d'une église paléochrétienne, elle-même construite sur l'emplacement d'un édifice antique qui pourrait bien être un sanctuaire de Thétis et des Néréides; cf. aussi A.J.B WACE, The Topography of Pelion and Magnesia, in JHS, 26 (1906), p. 143-168 (en particulier 145148); A. PHILIPPSON, Die griechischen Landschaften, Bd. I, Teil I, 1950, p. 161. Je relève que la région de Liri pourrait receler d'autres vestiges provenant d'un sanctuaire antique : un relief votif, représentant un orant face à deux divinités (Ascléplos et Hygie ?) a été encastré, peu après la seconde guerre mondiale, dans le mur d'une autre chapelle, consacrée à la Dormition de la Vierge.

5 EUR., Andromaque, 1265 (trad. Jean et Mayotte Bollack). 
Pélée :

O maîtresse, ô noble compagne de mon lit, enfant de Nérée je te salue. Ce que tu fais là est digne de toi, et les enfants issus de toi le méritent. Puisque tu me l'ordonnes, je mets fin, déesse, à mon deuil, et je m'en vais enterrer ce mort (Néoptolème, fils d'Achille), puis me rendre dans les replis du Pélion, là même où j'ai pris dans mes bras ton corps superbe...

(Euripide, Andromaque, 1231-1232; 1254-1262; 1265-1278, trad. Jean et Mayotte Bollack ${ }^{6}$ )

Dans une pièce non identifiée, le même Euripide ${ }^{7}$ précise que la seiche fut la dernière métamorphose effectuée par Thétis poursuivie par Pélée, et que c'est bien sous cette forme qu'elle se laissa maîtriser et qu'elle subit l'étreinte (grâce aux conseils donnés à Pélée par un célèbre ressortissant de la contrée, le centaure Chiron, futur éducateur d'Achille ${ }^{8}$ ). Euripide toujours, dans son Iphigénie en Aulide (700 sq.), localise les noces mélusiniennes ${ }^{9}$ de Pélée et de Thétis, occasion du grand festin qui réunit les dieux, sur les saints contreforts du Pélion et non dans la région de Pharsale, éloignée de la mer, où les auteurs plus anciens (Pindare en particulier, dans la huitième Istbmique ${ }^{10}$ et d'autres encore auxquels fait allusion Tzetzes, op.cit., commentaire au vers 175, p. 84 Scheer) la faisaient conduire, enlevée sur l'attelage de Pélée, dans une scène évoquant le rapt de Perséphone. Euripide conjoignait ainsi le motif de la capture et de l'union amoureuse (motif lié au cap Sépias) à celui des noces (motif lié au sanctuaire du Thétideion de Pharsale, solidaire d'une tradition épique remontant aux Chants cypriens).

Nous devons nos informations les plus précises sur la localisation du Sépias au récit que fait Hérodote de la tempête qui brisa sur les côtes du Pélion, en 480 avant J.-C., une partie de la flotte de Xerxès ${ }^{11}$.

6 Paris, Les Editions de Minuit, 1994.

7 Fr. 1093 Nauck, cité par le commentaire de TZETZES à LYCOPHRON, aux vers 175 et 178 de l'Alexandra, p. 85 et 88 Scheer. Il convient de lire le texte tel qu'il est amendé par M. MAYER, art. Thetis, in RE, VI A 1 (1936), col. 207.

8 Sur l'importance de Chiron dans la mythologie relative à Thétis, cf. Paula PHILIPPSON, Thessalische Mythologie, Zürich, 1944, p. 158.

9 L'expression est d'A. LESKY, Thalatta. Der Weg der Griechen zum Meer, Wien, 1947, p. 121.

10 Pour une analyse de la donnée épique et lyrique, cf. Laura M. SLATKIN, The Power of Tbetis. Allusion and Interpretation in the Iliad, Berkeley-Los Angeles-Oxford, 1991 (sur la 8e Isthmique, voir p. 70 sq.).

11 Sepias, chez Hérodote, désigne tantôt l'ensemble de la côte est de la presqu'île de Magnésie, tantôt le cap lui-même, en face de Skiathos, qui donne son nom à cette côte. Certains auteurs (encore suivis par D. MUELLER, Topographiscber Blldkommentar zu den Historten Herodots. Griechenland, Tübingen, 1987 , p. 360-364), n'ont pas reconnu ce double usage; ne comprenant pas, de ce fait, que Ja flotte de Xerxès, partie de Therme, puisse se trouver sur les rives du Sépias après un seul jour de navigation, ils se trouvèrent entraînés à identifier le cap Sépias avec l'actuel cap Puri, au nord du village de Chorefton. Hérodote précise pourtant bien que seule la violence 
Ce naufrage fut très avantageux à Aminocle, fils de Crétine, Magnète, qui avait du bien aux environs du promontoire Sépias. Quelques temps après il enleva quantité de vases d'or et d'argent que la mer avait jetés sur le rivage. Il trouva aussi des trésors des Perses, et se mit en possession d'une quantité immense d'or. Cet Aminocle devint très riche par ce moyen; mais il était d'ailleurs malheureux, car ses enfants avaient été tués, et il était vivement affligé de cette calamité

Ce petit apologue sur les contradictions de la fortune, et sur l'argent qui ne fait pas le bonheur, s'inscrit en marge du récit des événements qui précédèrent la fameuse bataille navale de l'Artémision. Prévoyant l'imminent déferlement des « barbares », les responsables grecs, s'étant concertés, avaient décidé de garder les deux passages inévitables des Thermopyles et de l'Artémision, que devaient emprunter, nécessairement et respectivement, les troupes terrestres et navales de Xerxes. L'Artémision, qui doit son nom au sanctuaire eubéen d'Artemis proseota ( tournée vers l'Orient ») désigne ici l'ensemble du détroit situé entre l'Eubée et la Magnésie; sur la côte sud de la presqu'île de Magnésie, cela correspond à la région qui s'étend du cap Sépias (en face de l'île de Skiathos), jusqu'à Trikeri et au port d'Hagia Kiriaki; c'est la côte, essentiellement sauvage et rocailleuse, qui sépare le golfe pagasétique (actuel golfe de Volos) de l'ouverture sur l'Égée. Ayant décidé de défendre ce passage, les Grecs procèdent, à Delphes, dans le temenos de Thyè fille du Céphise, à un sacrifice aux Vents (VII, 178). Une avant-garde d'élite de dix vaisseaux perses cingle vers Skiathos, où elle rencontre trois vaisseaux grecs envoyés en éclaireurs. Deux vaisseaux grecs sont pris, le troisième s'échoue et son équipage s'enfuit. La flotte stationnée dans l'Artémision apprend l'événement par des feux allumés sur l'île de Skiathos; une peur violente incite les Grecs à abandonner l'Artémision, et à se replier sur Chalcis, pour défendre l'Euripe tout en laissant des guetteurs sur les hauteurs de l'Eubée (VII, 179-182). Trois des dix vaisseaux orientaux dont nous venons de parler abordent alors l'îlot nommé Murmex (« la fourmi »), entre Skiathos et la Magnésie (c'est-à-dire à l'entrée du détroit de l'Artémision); les « Barbares » élèvent sur ce récif une colonne dont ils ont amené les pierres, une stelen litbou, qui servira de repère indiquant le passage au reste de la flotte, qui attend à Therme (non loin de l'actuelle Salonique). La flotte perse entreprend alors sa navigation en direction de Skiathos, le long de

extraordinaire de la tempête explique la dispersion des vaisseaux, du nord de la côte est, jusqu'au cap Sépias proprement dit (VII, 188, infra). Les données suggérées par Apollonios de Rhodes (supra, n. 3), elles, sont sujettes à caution (quand on remonte la côte en s'éloignant du cap Sépias, précisément situé tout près, et en face, de Skiathos, on ne peut pas voir cette dernière " apparaître " au milieu de la mer, mais tout au plus " disparaitre »). P.W. WALLACE, Aphetai and the Battle of Artemision, in Studies presented to Sterling Dow on bis Eigbtietb Birtbday, Durham, 1984, p. 305310, parle avec raison de la « garbled geography of Ap. Rhod. I.580-91» (p. 305, n. 2). Nous ne suivons toutefois pas WALLACE dans son hypothèse sur la localisation des Aphètes, et préférons nous en tenir à la position bien argumentée de C. HIGNETT, Xerxes' Invasion of Greece, Oxford, 1963 , p. $167-177$. 
la côte est de Magnésie. C'est un marin de Skyros engagé dans les troupes perses, Pammon (un familier de la région), qui le premier remarque et signale le repère érigé sur l'îlot de la Fourmi. L'immense flotte barbare se déploie sur la mer, au long des rives inhospitalières qui s'étendent de la ville de Casthanée (aux pieds de l'actuelle Zagora) jusqu'au promontoire du Sépias (VII, 183). La côte n'offre pas de mouillage suffisant pour toute cette armada (environ 1200 vaisseaux de guerre auxquels s'ajoutaient 3000 vaisseaux de transport, selon Hérodote). Le soir venu, après un jour de navigation, la plus grande partie de la flotte stationne donc au large (sur huit rangs de hauteur). À l'issue d'une nuit sereine et calme, la mer s'agite. Une tempête dominée par un vent d'est, nommé bellespontias par les gens de l'endroit, s'élève au point du jour :

Ceux qui s'aperçurent que le vent allait en augmentant, et qui étaient à la rade, prévinrent la tempête et se sauvèrent ainsi que leurs vaisseaux, en les tirant à terre. Quant à ceux que le vent surprit en pleine mer, les uns furent poussés contre ces endroits du mont Pélion qu'on appelle ipnes (fours) ${ }^{12}$, les autres contre le rivage; quelques-uns se brisèrent au promontoire Sépias; d'autres enfin à Casthanée; tant la tempête fut violente.

(Hérodote, VII, 188, trad, Larcher).

Environ 400 bateaux perses furent brisés ou coulés, et très nombreux furent les morts. Les Grecs, qui apprennent le désastre ennemi grâce aux guetteurs postés sur les sommets de l'Eubée, reprennent confiance et font revenir leur flotte de l'Euripe à l'Artémision, non sans offrir des libations à Poséidon « Sauveur ». On se souvient qu'ils avaient déjà sacrifié aux Vents, à Delphes. Poséïdon et les Vents représentent ainsi l'interprétation religieuse panhellénique. Les Athéniens, pour leur part, attribuent le bienfait à Borée, le Thrace, qui les a déjà aidés autrefois (en 492) en envoyant une première tempête contre les Perses de l'expédition de Mardonios, au large de l'Athos; ils se souviennent en outre qu'il est leur gendre, Borée ayant enlevé Orythie, fille de leur vieux roi Érechthée : ils élèveront donc au Vent divin, à l'issue de la guerre, un autel au bord de l'Ilissos (VII, 189; cf. le début du Phèdre de Platon).

À ces deux interprétations, l'une panhellénique, l'autre chauvine, fait contraste l'interprétation des «barbares», qui ne doit rien à la religion iranienne, mais qui est directement inspirée (selon Hérodote) par le contexte local du culte de Thétis dans la région du Sépias, à savoir un culte adressé à une divinité grecque des profondeurs de la mer, dont le fils (symbole épique de la lutte des Grecs contre les adversaires asiatiques) devient un seigneur divin des passages maritimes (Achille Pontarchès, dont la tombe est signalée par une

12 Il s'agit vraisemblablement de grottes marines, parfois abris des phoques, qui abondent dans cette région. Ces grottes peuvent présenter l'aspect de fours traditionnels, tels qu'on les rencontre encore en Thessalie : cf. D. MUELLER, op. cit. (n. 11), p. 329-332, avec ill. 4 p. 331. 
stèle sur les côtes de l'Hellespont, mais auquel un séjour immortel est néanmoins assuré dans l'île blanche du Pont-Euxin ${ }^{13}$ ) :

La tempête dura trois jours. Enfin les mages l'apaisèrent le quatrième jour en immolant des victimes à Thétis et aux Néréïdes... Ils offrirent des sacrifices à Thétis parce qu'ils avaient appris des Ioniens ${ }^{14}$ qu'elle avait été enlevée de ce canton-là même par Pélée, et que toute la côte de Sépias lui était consacrée, ainsi qu'au reste des Néréides. Quol qu'il en soit, le vent cessa le quatrième jour.

(Hérodote, VII, 191, trad. Larcher)

Peut-être faut-il se souvenir aussi, avec Gregory $\mathrm{Nagy}^{15}$, que le vent dont une partie de la flotte perse fut victime était appelé bellespontias, d'un nom le désignant comme issu des régions où repose l'immortel Achille. On peut ajouter qu'Achille est un Myrmidon descendant des " fourmis " (murmekes), peuple autrefois sorti de terre sur l'île d'Égine, et sur lequel régna d'abord Éaque, fondateur de la lignée royale qui conduit à Pélée. À ces « fourmis » ancestrales semble à son tour faire allusion, à la manière d'un présage passé inaperçu, le curieux signal (une stèle) de l'îlot dit « de la Fourmi », au large du Sépias, qui attire les Barbares vers les lieux de la catastrophe. Cette multiplicité

13 CF. G. NAGY, Le meilleur des Acbéens. La fabrique du béros dans la poéste grecque archalque, trad. de l'anglais par Jeannie Carlier et Nicole Loraux, Paris, Seuil, 1994, p. 392. Un texte étrange et raffiné de PHILOSTRATE (Heroicus, $208 \mathrm{~K}$ ) mérite de rejoindre le dossier; l'auteur imagine (ou rapporte?) que dans un lointain passé, alors que la Thessalie était encore gouvernée par les Éacides, l'oracle de Dodone avait ordonné un étrange rituel : parti de Thessalie, un bateau à voile noire cinglait, une fois par année, en direction de la Troade; à son bord, deux fois sept théores emportaient de chez eux tout ce qui était nécessaire à l'accomplissement du rite : un bovidé blanc et un bovidé noir, l'un et l'autre domestiques, du bois du Pélion, du feu, de l'eau du Spercheios; soucieux du moindre détail, et afin de n'avoir à utiliser que des matériaux issus de Thessalie, même au cas où le vent ralentirait leur voyage, jls emmenaient aussi des couronnes d'amarante, qui ne se fanent pas. Parvenus au large du tombeau d'Achille, obligatoirement de nuit (les Thessaliens se trouvent alors en terre ennemie), ils adressaient à Thétis, avant de toucher terre, un hymne l'invitant à venir assister au double sacrifice, héroïque et divin, qu'ils allaient pratiquer pour son fils (à la fois un mort, que retient la terre de Troie, et un dieu, localisé dans le Pont). Ayant accosté, ils dansaient en courant vers le tombeau, tout en faisant retentir leurs boucliers comme à la guerre et en appelant Achille de leurs clameurs. "Puis, ayant couronné le sommet de la colline et creusé à cet endroit des fosses sacrificielles, ils immolaient le bovin noir en lui tranchant la gorge, comme on le fait pour un mort. Ils appelaient aussi Patrocle, l'invitant au repas, faisant cela aussi en témoignage de reconnaissance pour Achille. Ayant pratiqué le rituel funèbre, ils s'en retournaient en direction du bateau et sacrifiaient le second bovin, à Achille toujours mais conformément cette fois au rituel divin, sur la plage, en se limitant toutefois aux rites préliminaires de la corbeille et des viscères. Ils remettaient aussitôt à la voile, en emmenant au large les viandes sacrificielles, ne désirant point festoyer en terre ennemie». Fruit d'une élaboration littéraire tributaire d'une pluralité de souvenirs classiques, ce texte témoigne, au IIe s. de notre ère, de l'importance pour les Anciens du réseau mythico-géographique qui relie, sous le signe d'Achille et de Thétis, la Thessalie à l'Hellespont.

14 On sait en effet que Thétis et les Néréides, ainsi qu'Achille, pouvaient faire l'objet d'un culte en Ionie : pour l'exemple d'Érythrées, cf. F. GRAF, Nordion/sche Kulte, Schweizerisches Institut in Rom, 1985, p. 351-353.

15 Loc.cit. 
d'allusions au mythe s'élève comme un commentaire immédiat, à chaud, pluriel, destiné à couvrir un événement hautement traumatisant. C'est en partie à cette réalité, du même coup, que l'on doit le prestige des mythes relatifs à la divinité du Sépias, et la justesse des vers d'Euripide qui lui sont consacrés.

Université de Genève

Philippe BORGEAUD

Faculté des Lettres

Département des Sciences de l'Antiquité

$\mathrm{CH}-1211$ GENÈVE 4 